ARTICLE

Received 18 Mar 2015 | Accepted 27 Jul 2015 | Published 14 Sep 2015

DOl: $10.1038 /$ ncomms 9176

OPEN

\title{
Structure of HCMV glycoprotein B in the postfusion conformation bound to a neutralizing human antibody
}

Sumana Chandramouli ${ }^{1}$, Claudio Ciferri ${ }^{1}{ }^{\dagger}$, Pavel A. Nikitin ${ }^{2}$, Stefano Caló ${ }^{3}$, Rachel Gerrein ${ }^{1}$, Kara Balabanis ${ }^{1}$, James Monroe ${ }^{1}$, Christy Hebner ${ }^{2}$, Anders E. Lilja ${ }^{1, \dagger}$, Ethan C. Settembre ${ }^{4} \&$ Andrea Carfi ${ }^{1}$

Human cytomegalovirus (HCMV) poses a significant threat to immunocompromised individuals and neonates infected in utero. Glycoprotein B ( $\mathrm{B} B$ ), the herpesvirus fusion protein, is a target for neutralizing antibodies and a vaccine candidate due to its indispensable role in infection. Here we show the crystal structure of the HCMV gB ectodomain bound to the Fab fragment of $1 \mathrm{G} 2$, a neutralizing human monoclonal antibody isolated from a seropositive subject. The $\mathrm{gB} / 1 \mathrm{G} 2$ interaction is dominated by aromatic residues in the $1 \mathrm{G} 2$ heavy chain CDR3 protruding into a hydrophobic cleft in the gB antigenic domain 5 (AD-5). Structural analysis and comparison with HSV gB suggest the location of additional neutralizing antibody binding sites on HCMV gB. Finally, immunoprecipitation experiments reveal that $1 \mathrm{G} 2$ can bind to $\mathrm{HCMV}$ virion $\mathrm{gB}$ suggesting that its epitope is exposed and accessible on the virus surface. Our data will support the development of vaccines and therapeutic antibodies against HCMV infection.

\footnotetext{
${ }^{1}$ GSK Vaccines, 45 Sidney Street, Cambridge, Massachusetts 02139, USA. ${ }^{2}$ Novartis Institutes for Biomedical Research, Emeryville, California 94608, USA. ${ }^{3}$ GSK Vaccines, Via Fiorentina 1, Siena 53100, Italy. ${ }^{4}$ Novartis Influenza Vaccines, 45 Sidney Street, Cambridge, Massachusetts 02139, USA.

$\dagger$ Present addresses: Department of Structural Biology, Genentech Inc., 94080 South San Francisco, California, USA (C.C.); Hookipa Biotech AG, 1030 Vienna, Austria (A.E.L.). Correspondence and requests for materials should be addressed to S.Ch. (email: sumana.x.chandramouli@gsk.com) or to A.C. (email: andrea.x.carfi@gsk.com).
} 
uman cytomegalovirus (HCMV) is a double stranded DNA virus of the $\beta$-herpesvirus family. HCMV is the leading cause of congenital and neonatal hearing loss resulting from vertical virus transmission following infection or reactivation of latent virus in pregnant women ${ }^{1}$. In addition, HCMV is one of the most common opportunistic pathogens affecting immunosuppressed transplant patients. Infection of seronegative subjects receiving solid organ transplants from seropositive donors is positively correlated with increased incidence of allograft rejection ${ }^{2}$. Currently, antiviral therapy following transplantation remains the only mode of intervention, often combined with hyperimmune globulin transfusion to help control viremia ${ }^{3}$. Though development of a vaccine against HCMV has been listed as a top priority by the Institute of Medicine $e^{4,5}$, none has been licensed so far.

The HCMV genome encodes several envelope glycoproteins. Among them, glycoprotein $\mathrm{B}(\mathrm{gB})$ and the $\mathrm{gH} / \mathrm{gL}$ heterodimer are conserved within the herpesvirus families $(\alpha, \beta$ and $\gamma)$ forming the core fusion machinery essential for viral entry into cells ${ }^{6}$. HCMV cell entry can be distinguished into two steps, receptor binding and membrane fusion. Receptor-binding function resides within distinct $\mathrm{gH} / \mathrm{gL}$-containing complexes: $\mathrm{gH} / \mathrm{gL} / \mathrm{gO}$ for entry into fibroblasts and $\mathrm{gH} / \mathrm{gL} / \mathrm{UL} 128 / \mathrm{UL} 130 / \mathrm{UL} 131 \mathrm{~A}$ (Pentamer) for entry into epithelial/endothelial cells ${ }^{7-10}$. Membrane fusion is mediated by $\mathrm{gB}$, which acts as the fusogen ${ }^{11,12}$. How cognate receptor binding of $\mathrm{gH} / \mathrm{gL}$ complexes triggers the conformational changes in $\mathrm{gB}$ required for membrane fusion remains unknown. In the case of herpes simplex virus (HSV), an $\alpha$-herpesvirus, bimolecular complementation assays have shown evidence for an interaction between $\mathrm{gB}$ and $\mathrm{gH} / \mathrm{gL}$ during cell fusion ${ }^{13,14}$. Preliminary co-immunoprecipitation experiments in epithelial cells have also provided some evidence for an interaction between $\mathrm{gH} / \mathrm{gL}$ and $\mathrm{gB}$ in $\mathrm{HCMV}^{15}$.

HCMV subunit vaccines incorporating soluble forms of $\mathrm{gB}$ have been under development for several years (reviewed in ${ }^{16,17}$ ). Immunization with a gB subunit adjuvanted with MF59 resulted in $\sim 50 \%$ protective efficacy against primary infection in postpartum healthy women ${ }^{18,19}$. Similarly, an MF59-adjuvanted gB vaccine led to significant reduction in viremia and shortened antiviral treatment in patients receiving solid organ transplants ${ }^{20}$. Furthermore, in a guinea pig maternal immunization/infection model, a three-dose regimen with a guinea pig CMV gB subunit vaccine adjuvanted with AS01 or AS02 reduced pup mortality by $64-84 \%$, and reduced viremia in infected dams by nearly sixfold $^{21}$. Together these studies confirmed that the gB subunit vaccines were safe and immunogenic, though further improvements in potency and durability of protection were desirable.

Monoclonal antibodies (mAbs) against HCMV have a potential use as an alternative or addition to the current forms of HCMV therapy. Neutralizing antibodies against HCMV gB were shown to inhibit entry of cell-free virus in several cell types (including fibroblasts, endothelial and epithelial cells) and to prevent cell-to-cell spread ${ }^{22}$. Moreover in a recent study, protection from brain pathology in murine cytomegalovirus infected mice was accomplished by passive transfer of a gB-specific monoclonal antibody ${ }^{23}$. A better understanding of the antigenic determinants of $\mathrm{gB}$ and biochemical and functional characterization of potent, broadly neutralizing antibodies is invaluable for developing therapeutic antibody candidates and vaccines against HCMV infection.

Crystal structures of HSV and Epstein-Barr Virus (EBV) postfusion $\mathrm{gB}$ have shown a close similarity despite the relatively low sequence identity ${ }^{11,24}$. These structures revealed that the gB ectodomain has an elongated three-lobed shape and is composed of five structural domains (I-V). In addition, low resolution negative stain electron microscopy (EM) studies of HCMV gB have confirmed a conserved overall architecture for postfusion $\mathrm{gB}$ across the three herpesvirus families ${ }^{25-27}$. Finally, structural analysis has revealed a close similarity between herpesvirus $\mathrm{gB}$ and the postfusion conformation of the vesicular stomatitis virus $\mathrm{G}$ and the baculovirus Gp64 glycoproteins ${ }^{28-30}$ suggesting that recombinant $\mathrm{gB}$ spontaneously folds into the postfusion conformation. Despite this progress, the crystal structure of HCMV gB has remained elusive, in part due to the difficulty of obtaining large amounts of homogeneous sample.

EM and mutagenesis studies have located some of the key neutralizing sites in HSV $\mathrm{gB}^{11,31}$. In HCMV gB, five antigenic domains ( $\mathrm{AD}$ ) have been defined, with neutralizing epitopes mapped to AD-1 (within structural domain IV), AD-2 (within the first $80 \mathrm{~N}$-terminal residues), AD-4 (in structural domain II) and AD-5 (in structural domain $\mathrm{I})^{32,33}$. AD-3 resides in the very $\mathrm{C}$ terminus of $\mathrm{gB}$ beyond the transmembrane region and no neutralizing antibodies targeting this domain have been reported to date. Many human neutralizing antibodies targeting gB have been isolated from IgG-secreting memory B cells from HCMV seropositive individuals ${ }^{33,34}$ and shown to neutralize infection of both fibroblasts and epithelial cells in the absence of complement. Several of these antibodies have been found to target AD-4 (SM1-6, SM3-1, SM4-5, SM5-1, SM6-5 and SM11-17) and AD-5 (SM10, 1G2, SM12 and 2C2), and key gB residues critical for binding to mAbs have been identified ${ }^{35,36}$. The structure of a complex between the SM5 Fab and an E.coli-expressed gB AD-4 domain only (spanning residues $112-132$ and 344-438) has also recently been reported ${ }^{37}$.

We have introduced mutations in HCMV gB that allow its secretion as a homogeneous and stable postfusion trimer from mammalian cells. Here we report the crystal structure of this molecule in complex with the 1G2 Fab. The structure provides the first atomic view of HCMV gB and of its interaction with a broadly neutralizing antibody targeting the conserved $\mathrm{AD}-5$ site. These data will guide the design of improved gB-based HCMV vaccines and will support the development of therapeutic antibodies against this medically relevant human pathogen.

\section{Results}

Generation of a soluble and homogeneous gB ectodomain. The ectodomain of HCMV gB (Merlin strain), residues 1-698, with a 6-His tag at the $\mathrm{C}$ terminus was expressed in HEK293S $\mathrm{GnTI}^{-}$cells (Fig. 1a). To increase protein secretion, we mutated three hydrophobic residues in the fusion loops with the corresponding more hydrophilic amino acids from HSV-1 gB (I157H, H158R and W240R). We also mutated the canonical furin cleavage site to decrease protein heterogeneity caused by incomplete processing during expression, as well as the exposed Cys246 (Cys246Ser) to prevent formation of spurious disulfide bonds (Fig. 1a). Despite these changes, size exclusion chromatography (SEC) and negative stain EM (Fig. 1b) revealed that the protein (gB-698) formed dimers of the characteristic three-lobed postfusion trimers ${ }^{26,38}$. Analysis of the EM images suggested that dimerization was mediated by the base of the $\mathrm{gB}$ trimer, presumably due to intrinsic surface hydrophobicity. Thus, we introduced a glycosylation site in fusion loop-2 (Trp240Asn and Tyr242Thr), predicted to be solvent exposed in the trimer, to interfere with dimerization. EM and SEC confirmed that this construct, gB-698glyco, does not dimerize even at high protein concentration (Fig. 1b).

EM of HCMV gB bound to 1G2 Fab. EM was used to initially characterize the $g B / 1 G 2$ Fab complex. Comparison of negative stain two-dimensional class averages of gB-698glyco alone and 

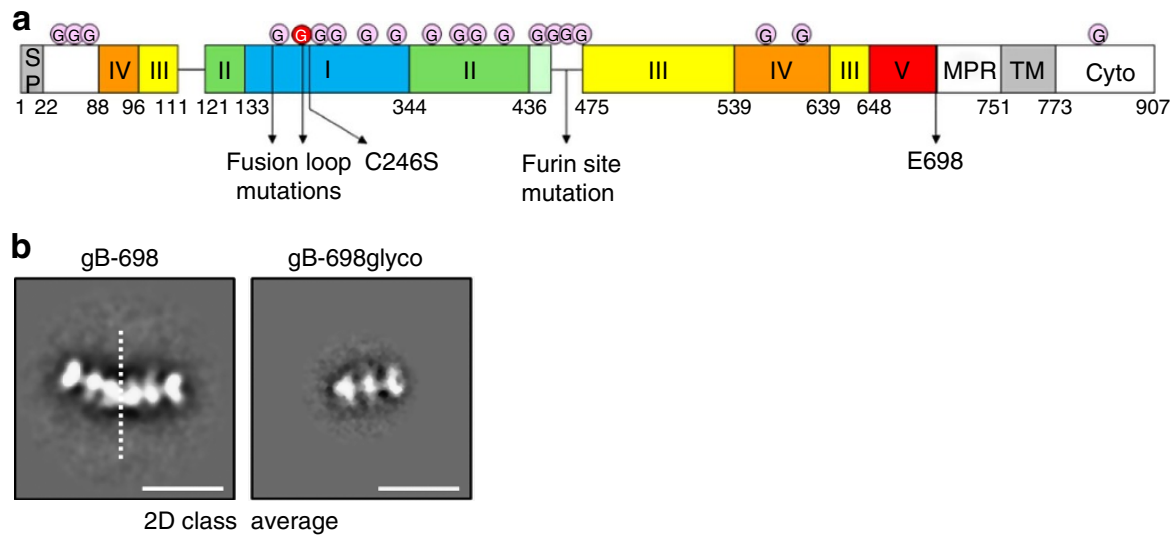

Figure 1 | Electron microscopy of gB ectodomain constructs. (a) Schematic of full-length $\mathrm{gB}$ with domains I-V coloured as in ${ }^{11}$. Mutations introduced in gB ectodomain constructs and glycosylation sites are indicated by arrows and pink circles, respectively. An additional glycosylation site introduced in fusion loop 2 is indicated by a red circle. SP, signal peptide; MPR, membrane proximal region; TM, transmembrane region; Cyto, cytoplasmic domain. E698 indicates the last $\mathrm{C}$-terminal residue in the $\mathrm{gB}$ ectodomain constructs. Details of mutations in the fusion loops and furin site are reported in Materials and Methods. (b) Two-dimensional class averages of negative stain electron micrographs of gB-698 (left panel) and gB-698glyco (right panel). Images represent projections of molecule side views. Dimer boundary (left panel) is indicated by a dashed line. Scale bar, $200 \AA$.

a
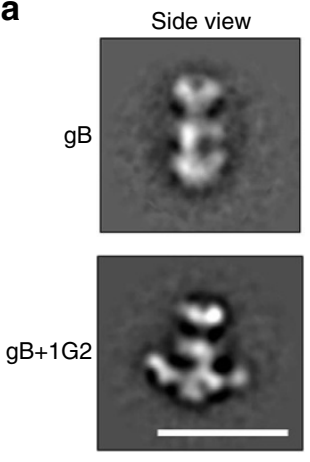

C

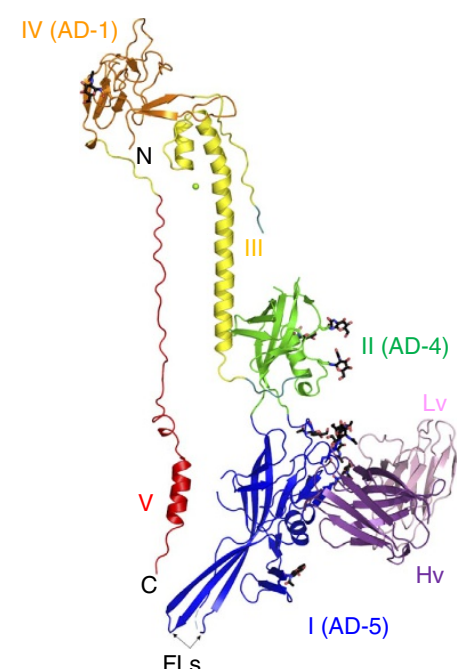

b
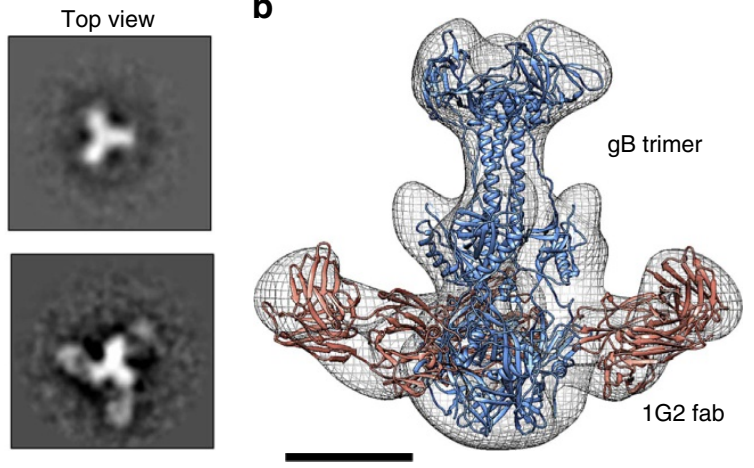

d

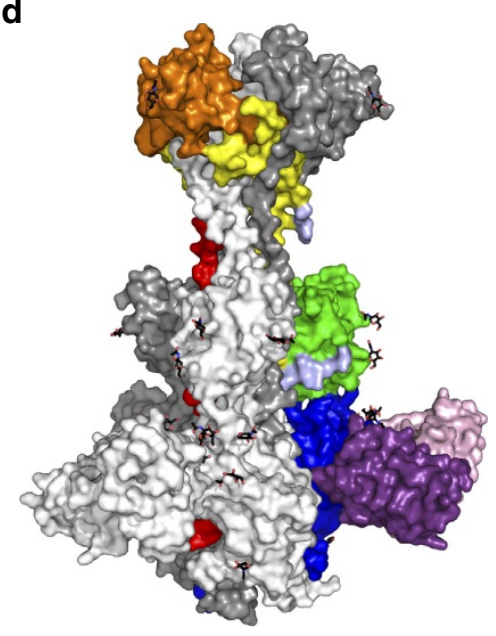

Figure 2 | Structure of $\mathbf{g B / 1 G 2}$ complex. (a) EM two-dimensional class averages of gB-698glyco (top panels) and gB-698glyco in complex with $1 \mathrm{G} 2 \mathrm{Fab}$ (bottom panels). Scale bar, $200 \AA$. (b) Low resolution three-dimensional reconstruction of gB-698glyco/1G2 Fab complex generated from EM data with homology models of $\mathrm{gB}$ and $1 \mathrm{G} 2$ fitted in the electron density map. gB trimer is shown in blue and the $1 \mathrm{G} 2$ Fab is shown in red. Scale bar, $50 \AA$. (c) Crystal structure of $\triangle \mathrm{NgB}$-glyco/1G2 complex (PDB ID: 5C6T). The asymmetric unit contained a single gB monomer bound to 1G2. The domains of $g B$ are coloured as in Fig. 1a. The 1G2 Fab heavy chain is shown in purple and the light chain in light pink. The glycans resolved in the structure are shown in black, and a Mg ion in domain III is shown as a green dot. (d) Surface representation of the $\Delta \mathrm{NgB}-\mathrm{glyco} / 1 \mathrm{G} 2$ trimer. One monomer is positioned and coloured as in (c) while the other two are represented in white and grey. 
bound to $1 \mathrm{G} 2$ confirmed that all three sites on the gB trimer are occupied by Fab molecules (Fig. 2a and Supplementary Fig. 1A). Moreover, individual particles, extracted from negative-stained micrographs, were used to generate a low resolution threedimensional reconstruction of the complex at $19 \AA$, using the crystal structure of HSV gB low pass filtered at $60 \AA$ as initial model. In agreement with previous mutagenesis data ${ }^{33,36}$, the EM analysis confirmed that $1 \mathrm{G} 2$ binds to structural domain I (residues 134-343; Figs $1 \mathrm{a}$ and 2b).

Structure determination of $\mathrm{gB} / 1 \mathrm{G} 2$ Fab complex. Initial attempts at crystallizing gB-698glyco alone or in complex with 1G2 Fab were unsuccessful. We therefore enzymatically deglycosylated the protein with endoglycosidase $\mathrm{H}$ and performed in situ limited proteolysis with subtilisin $\mathrm{E}$ to remove flexible regions that could interfere with crystallization ${ }^{39,40}$. This treatment resulted in crystals that diffracted to $4.3 \AA$ resolution. Subsequently, we deleted the first $63 \mathrm{~N}$-terminal residues, shown to be flexible in HSV-1 $\mathrm{gB}^{11}$, from gB-698glyco resulting in the $\Delta \mathrm{NgB}$-glyco construct (residues 86-698). The deglycosylated $\Delta$ NgB-glyco/1G2 Fab complex crystallized readily without need for protease treatment. After screening several crystals, a 3.6- $\AA$ resolution dataset was obtained and the structure determined by the molecular replacement method (Table 1). The resulting electron density maps allowed model building for $\mathrm{gB}$ and the $\mathrm{Fv}$ portion of the Fab (Supplementary Fig. 2). Though crystal packing allows enough space for the constant regions of the Fab heavy and light chains, no electron density was observed for these

Table 1 | X-ray data collection and refinement statistics.

$\triangle \mathrm{NgB}-\mathrm{glyco}+1 \mathrm{G} 2 \mathrm{Fab}$

Data collection

Space group

Cell dimensions

$a, b, c(\AA)$

$\alpha, \beta, \gamma\left({ }^{\circ}\right)$

Resolution $(\AA)$

No. of reflections

No. of unique reflections

$R_{\text {merge }}$

$|/ \sigma|$

Completeness (\%)

Redundancy

$\mathrm{P} 2{ }_{1} 3$

$176.49,176.49,176.49$ $90.0,90.0,90.0$

$50-3.55(3.74-3.55)^{\star}$

$1,56,458(22,493)$

$22,423(3,233)$

$0.104(1.061)$

$16.4(2.1)$

$100(100)$

$7.0(7.0)$

Refinement

Resolution $(\AA)$

No. reflections

$R_{\text {work }} / R_{\text {free }}$

No. atoms

Protein

Ligand/ion

Water

B-factors

Protein

Ligand/ion

Water

$19.983-3.6$

21,341

$0.212 / 0.260$

6,270

141

$-$

116

180

R.m.s. deviations

Bond lengths ( $A$ )

Bond angles $\left({ }^{\circ}\right)$

Ramachandran plot

Favoured

Allowed

Outliers

0.005

1.147

$85.8 \%$

$11.5 \%$

$2.7 \%$

R.m.s. deviation, root-mean-square deviation

*Values in parentheses are for highest-resolution shell.

$\dagger$ Measured using Molprobity. even at the end of refinement, suggesting that they are flexibly linked to the variable region and adopt different orientations in the crystals.

HCMV gB structural analysis and comparison with related gBs. The overall domain organization of postfusion HCMV $\mathrm{gB}$ is similar to HSV and EBV gBs. Five structural domains are distributed across the length of the molecule (Fig. 2c,d), with structural domains I and II adopting PH-domain-like folds. Domain I also harbours bipartite hydrophobic fusion loops exposed at the base of the molecule. Domain III forms a long central coiled-coil helix leading up to domain IV, the crown at the top of the molecule. Domain V, composed of the very C-terminal residues of the ectodomain, zips down the length of the molecule nestled between the other two protomers of the trimer, terminating close to the fusion loops at the base of the molecule.

The sequence of HCMV gB shares roughly 30\% identity with both HSV and EBV gBs. Taken individually, each of the HCMV gB structural domains superpose well with their counterparts in HSV and EBV (Supplementary Fig. 3). RMSD values range from $0.85 \AA$ for the central helix to $2.96 \AA$ for domain I (Supplementary Table 1) with most local differences occurring in loops. Domain II also very closely overlaps with the crystal structure of the isolated bacterially expressed domain II bound to SM5 Fab ${ }^{37}$, with an RMSD of $0.75 \AA$. However, when the three trimers are superimposed by aligning their highly conserved central helix, significant twists become apparent in the relative location of domains I, II and IV (Fig. 3a,b) with the HCMV gB structure occupying an intermediate position between HSV and EBV gBs. Also in the context of the trimer, within domain I, the $\beta 7-\beta 8$ loop of protomer $A$ sits adjacent to $\beta 4, \beta 5$ and $\beta 11$ of protomer $B$ (that contribute to the fusion loops) and the $\alpha \mathrm{F}$ helix of protomer $\mathrm{C}$ (Fig. 3c,d). This region differs between HSV and EBV gBs, with the $\beta 7-\beta 8$ loop occupying different positions to accommodate the exiting C-terminal tail ${ }^{24}$. This loop aligns better to the corresponding one from EBV gB (Fig. 3d), suggesting that the HCMV gB C-terminal tail, not present in the crystallized molecule, may follow a path similar to that of EBV $\mathrm{gB}$.

Larger differences are seen in domain IV (crown) of gB, which is composed predominantly of $\beta$-sheets with a fold unique to herpesvirus $\mathrm{gBs}^{11}$. In a superimposition of the domain IV of HCMV and HSV gB based on $\beta$-strands $\beta 33-35$, whose location is conserved in the two molecules, an inward rotation of the rest of the domain ( $\beta 1$ and $\beta 26-31)$ becomes apparent (Fig. 3e). This leads to formation of a smaller pore along the three-fold axis for HCMV gB compared with HSV gB (Fig. 3f). This domain is largely disordered in $\mathrm{EBV} \mathrm{gB}$, suggesting conformational flexibility of this region.

gB glycosylation sites and surface charge distribution. HCMV gB has a larger number of $N$-linked glycosylation sites than the other herpesvirus $\mathrm{gB}$ proteins, with 18 predicted in the primary sequence of the wild-type (WT) ectodomain (Fig. 1a) compared with five and nine for HSV and EBV gBs, respectively. All the glycosylation sites are conserved across HCMV strains with the only differences occurring in the proximity of the furin cleavage site and in the flexible $\mathrm{N}$ terminus. Electron density was observed for glycosylation of 10 of the potential 16 sites in the $\Delta \mathrm{NgB}$-glyco crystal structure, with the remaining six sites occurring in disordered regions of the structure. The surface of $\mathrm{gB}$ between domain I and II (AD-4 and AD-5, respectively) is particularly heavily $N$-glycosylated with seven glycosylation sites between them (that is, positions 281, 286, 302 and 341 in domain I and 383, 405 and 417 in domain II). These domains have been implicated in interaction with $\mathrm{gH} / \mathrm{gL}$ and the cell membrane in 

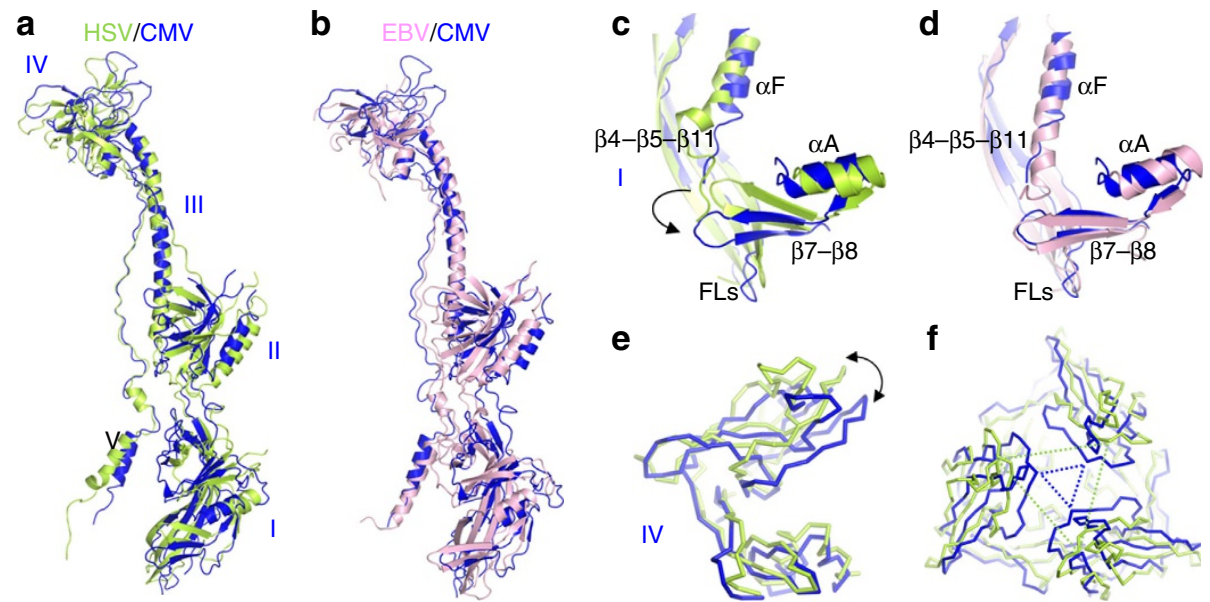

Figure 3 | Comparison of HCMV and other herpesvirus $\mathbf{g B s}$. HCMV gB is shown in blue, HSV-1 gB (PDB ID 2GUM ${ }^{11}$ ) in green and EBV gB (PDB ID 3FVC ${ }^{24}$ ) in pink. (a,b) Superimposition of HCMV gB and HSV gB (a) or EBV gB (b) based on alignment of the central helix (domain III). $(\mathbf{c}, \mathbf{d})$ Superimposition of domain I of HCMV and HSV gB (c) or EBV gB (d) shows that $\beta$-sheets 7 and 8 , and the $C$-terminal alpha helix occupy positions more similar to EBV gB than HSV gB. The $\beta$-sheets 4, 5 and 11 contributing to the fusion loops are shown in the background. (e) Side view of a superimposition of domain IV of HCMV and HSV gB based on the positionally conserved $\beta$-sheets 33-35 at the base. (f) Top view of (e) showing the smaller pore in the HCMV gB trimer compared with HSV gB. The location of Glu610 in HCMV gB and its homologous Glu631 in HSV gB are indicated by triangles in blue and green, respectively. All superimpositions were carried out using LSQKAB in CCP4.
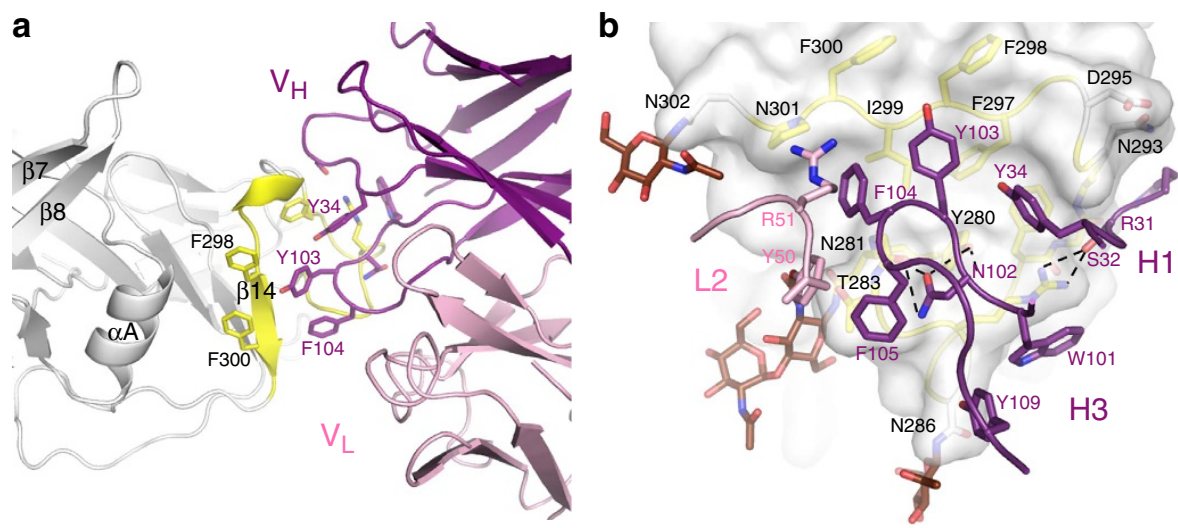

Figure 4 | The $\mathbf{g B / 1 G 2}$ interface. (a) Overall view of the gB/1G2 interface. gB domain I is shown in white with residues contacted by $1 G 2$ in yellow. The most important residues involved in the interaction are shown in stick representation. The view is rotated $180^{\circ}$ from the image in Fig. $2 \mathrm{c}$ for better clarity. (b) Details of the $\mathrm{gB} / 1 \mathrm{G} 2$ interaction with $\mathrm{gB}$ surface and key residues in stick representation. Only portions of HCDR1, HCDR3 and LCDR2 of 1G2 are shown. Hydrogen bonds between $\mathrm{gB}$ and the Fab are shown as dashed lines. Residue numbers for $1 \mathrm{G} 2$ heavy and light chains are shown in purple and pink, respectively. gB residues not forming direct contacts with 1G2 are shown in white. Glycosylated Asn residues at positions 281, 286 and 302 surrounding the epitope are shown with the linked NAGs in brown.

HSV $\mathrm{gB}^{13,41}$, and heavy glycosylation in their proximity may have evolved to mask these functional sites from the host antibody response.

The overall surface charge distribution on HCMV gB is distinct from that seen with HSV or EBV gB ${ }^{24}$ (Supplementary Fig. 4). This difference is most apparent in the crown, which has a positive patch unique to HCMV gB contributed by residues Arg563 and Lys634, enclosing a negatively charged inner groove towards the trimeric axis. At the base of the molecule, the fusion loops enclose a highly positively charged central groove, reminiscent of $\mathrm{EBV} \mathrm{gB}$. It has been proposed that the positive charge in this region may play a role in the interaction of $\mathrm{gB}$ with negatively charged phospholipid heads when the fusion loops insert into the membrane ${ }^{24}$.

gB/1G2 interface. 1G2 binds to a region of domain I (AD-5) encompassing a hydrophobic patch distinct from the fusion loops. Notably, 1G2 contacts gB mainly using heavy chain CDR1 and -3 (HCDR1 and -3 ), and establishes only limited interactions through the light chain CDR-2 (LCDR2; Fig. 4a and Supplementary Table 2). Over $1,350 \AA^{2}$ of surface area is buried in the complex, which is well within the range of $1,200-2,000 \AA^{2}$ observed for other antigen-antibody complexes ${ }^{2}$. The large buried surface area and surface complementarity of the $\mathrm{gB} / 1 \mathrm{G} 2$ interface $\left(S_{c}=0.637 ; 0.64-0.68\right.$ is the range for most antigenntibody complexes) are consistent with the tight binding affinity of the complex with a $K_{\mathrm{D}}$ of $0.17 \mathrm{nM}$ as measured by surface plasmon resonance (SPR) (Table 2).

Both hydrophobic and polar contacts contribute to the binding of gB to 1G2 (Fig. 4b and Supplementary Table 2). The aromatic side chains of HCDR3, Tyr103, Phe104 and Phe105 along with Asn102 and HCDR1's Tyr34 fill a hydrophobic cleft on the surface of $\mathrm{gB}$ consisting of residues Tyr280 and the stretch between Phe290 and Phe300. In addition, the binding of 1G2 seemingly leads to a kink in the $\mathrm{gB}$ backbone allowing the side 


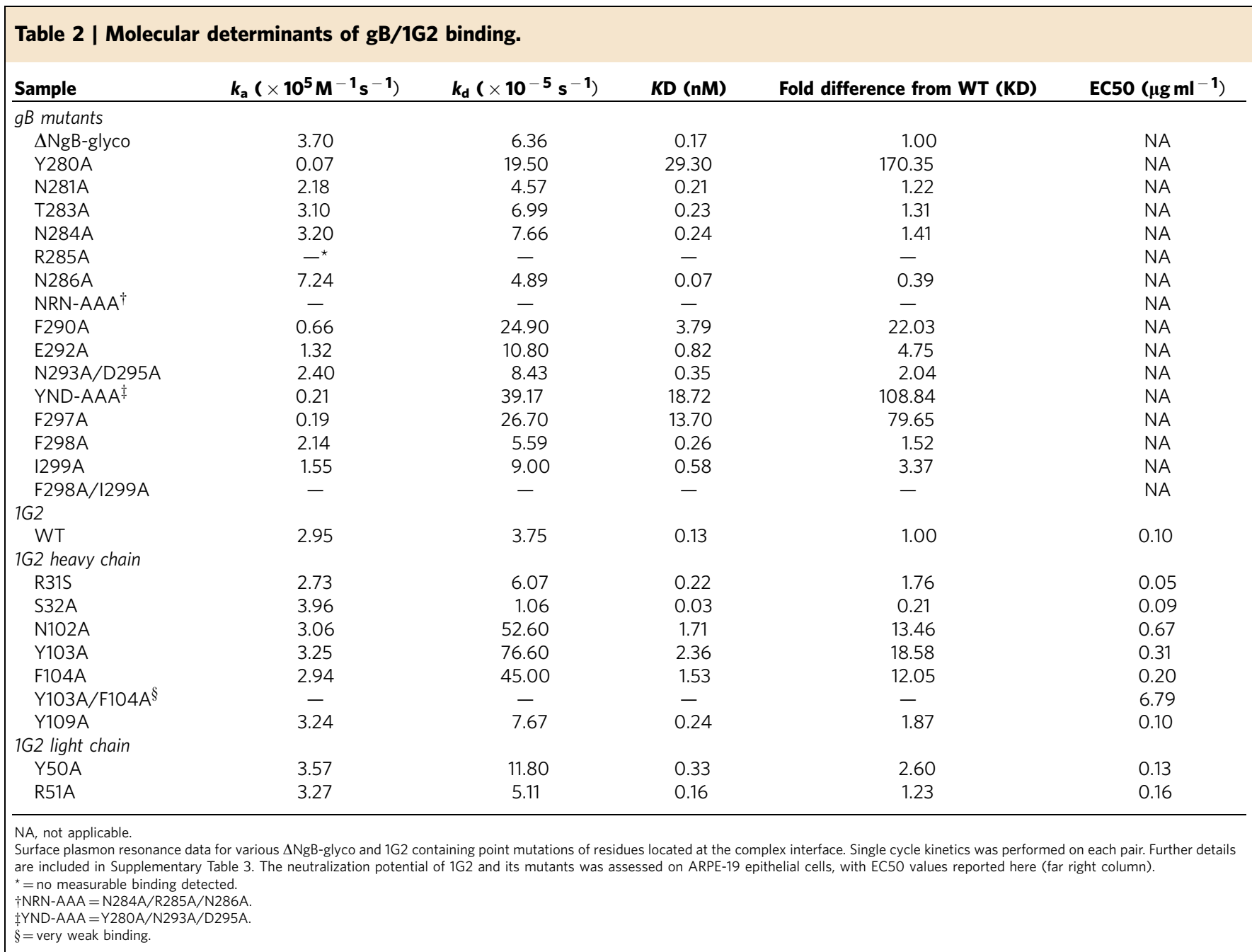

chain of Arg285 to stack against the indole ring of HCDR3 Trp101 (Fig. 4b). HCDR1 residues Arg31 and Ser32 make polar contacts with the $\mathrm{gB}$ main chain atoms between Phe290 and Ala294. In addition, Ser32 forms polar contacts with the side chain of gB Arg285. The 1G2 light chain appears to have a more limited role in the interaction with $\mathrm{gB}$. The side chain-OH groups of LCDR2 Tyr50 and gB Thr283 are $3.5 \AA$ apart and may form a water-mediated hydrogen bond. Furthermore, together with LCRD2 Arg51, Tyr50 contributes to the positioning of HCDR3 (Fig. 4b).

Molecular determinants of the $\mathrm{gB} / 1 \mathrm{G} 2$ interaction. To support the structural findings and gain additional insights into the $\mathrm{gB} / 1 \mathrm{G} 2$ interaction, we first mutated $\mathrm{gB}$ residues that are in proximity or part of the binding interface and measured their effect on the interaction using SPR (Table 2). The Arg285Ala mutation completely abolishes binding to $1 \mathrm{G} 2$. This result is in agreement with the extensive contacts established by the side chain of Arg285 with 1G2 (Fig. 4b and Supplementary Table 2). A number of other $\mathrm{gB}$ residues at the interface with $1 \mathrm{G} 2$ also contribute to complex formation. Point mutations of Glu292 or of hydrophobic residues Phe290, Phe297 and Ile299 to Ala significantly compromise the kinetics of the interaction, with the double mutant $\Delta \mathrm{NgB}$-glyco Phe298Ala/Ile299Ala having almost no binding to $1 \mathrm{G} 2$ (Table 2).

Of note, single point mutations of Tyr280 or Asn284 to Ala were previously shown to abrogate gB binding to $1 \mathrm{G} 2$ (ref. 36).
Also, mutating Tyr280 affected the binding of gB to SM10 and $2 \mathrm{C} 2$. The structure reveals that in addition to contacting 1G2 (Fig. 4b), Tyr280 forms hydrogen bonds with both exposed and buried residues of $\mathrm{gB}$, suggesting that it plays a role in preserving the structural integrity of the epitope. Consistent with this role, the Tyr280Ala mutation significantly reduced binding of 1G2 to $\triangle \mathrm{NgB}$-glyco as measured by SPR (Table 2 and Supplementary Table 3). While the Asn284Ala mutation was previously shown to reduce binding to $1 \mathrm{G} 2$ in an enzyme-linked immunosorbent assay (ELISA) ${ }^{36}$, the effect on the interaction was more subtle in our SPR experiments, and similar to three other mutations (Asn281Ala, Thr283Ala and Phe298Ala). Finally, Asn293 and Asp295, two residues that are critical for binding of $\mathrm{gB}$ to two other AD-5 specific mAbs SM10 and 2C2 (ref. 36), are in close proximity of the 1G2 epitope (Fig. 4b). However, these residues do not form direct contacts with $1 \mathrm{G} 2$ and when mutated to Ala, have minimal effect on 1G2 binding to gB (Table 2).

In contrast, mutation of Asn286 to Ala increased the overall gB/1G2 affinity (Table 2 and Supplementary Table 3). Interestingly, a recombinant virus carrying the same mutation was found to be more sensitive to neutralization by $1 \mathrm{G} 2$ (ref. 36). This residue falls within a canonical glycosylation site $\left({ }^{286} \mathrm{NAS}^{288}\right)$ and is exposed and glycosylated in the gB/1G2 structure (Fig. 4b). The absence of this glycan is expected to increase accessibility of $1 \mathrm{G} 2$ to its epitope resulting in tighter binding and, subsequently, higher neutralization potency.

To identify residues in $1 \mathrm{G} 2$ that are critical for $\mathrm{gB}$ binding and neutralization, we generated a panel of nine $1 \mathrm{G} 2$ variants carrying 


\begin{tabular}{|c|c|c|c|}
\hline \multirow[t]{2}{*}{ HCMV virus strain } & \multicolumn{2}{|c|}{$\begin{array}{l}\text { Neutralization EC50s } \\
\text { for } 1 \mathrm{G} 2\left(\mu \mathrm{gl}^{-1}\right)\end{array}$} & \multirow[t]{2}{*}{$\begin{array}{l}\text { Residue at } \\
\text { position } 28\end{array}$} \\
\hline & ARPE & NHDF & \\
\hline VR1814 (1G2 mAb) & 0.213 & 0.077 & Thr \\
\hline VR1814 (1G2 Fab) & 0.151 & NA & Thr \\
\hline TM-31354 & 0.230 & 0.411 & Thr \\
\hline 8816 & 0.046 & NA & Ala \\
\hline 8818 & 0.688 & 0.289 & Thr \\
\hline 8819 & 0.485 & 0.850 & Ala \\
\hline 8821 & 0.108 & 0.401 & Thr \\
\hline 8824 & 0.019 & 0.043 & Ala \\
\hline 8822 & 0.319 & 0.485 & Ala \\
\hline $\mathrm{TR}$ & 0.248 & 0.303 & Ala \\
\hline TM-28175 & NA & 0.138 & Ala \\
\hline MP-MD-805 & NA & 0.115 & Thr \\
\hline MP-LW-1802 & 0.104 & NA & Thr \\
\hline
\end{tabular}

HCMV, human cytomegalovirus; NA, not applicable; NHDF, normal human dermal fibroblasts. EC50 values for various clinical HCMV isolates measured on epithelial cells (ARPE-19) and fibroblasts (NHDF). Infection of $50 \%$ cells was determined based on HCMV IE expression. NA, clinical isolate did not express IE on a particular cell type.

point mutations of CDR residues located in the $\mathrm{gB} / 1 \mathrm{G} 2$ interface (Fig. 4b and Supplementary Table 2). Mutation of 1G2 HCDR3 Asn102, Tyr103 and Phe104 had the most profound effect on binding (Table 2). Substituting any of these residues with Ala increased the $K_{D}$ by an order of magnitude, mainly due to a faster off rate. In particular, a Tyr103Ala/Phe104Ala double mutant was severely compromised in its ability to bind $\Delta \mathrm{NgB}$-glyco with only very weak binding detected. By contrast, point mutation of residues further away from the interface (HCDR3 Tyr109Ala, HCDR1 Arg31Ser, LCDR2 Tyr50Ala or Arg51Ala) did not have any significant effect on the interaction. Surprisingly, the HCDR1 Ser32Ala mutation enhanced binding. It is possible that removing the polar side chain may allow the backbone carbonyl of residue 32 to interact with Arg285 without interference from the side chain hydroxyl of a Ser.

We next tested the $1 \mathrm{G} 2$ mutants in a virus neutralization assay. ARPE-19 epithelial cells were infected with HCMV (TB40/E strain) in the presence of WT $1 \mathrm{G} 2$ or its various mutants (Table 2). In line with the SPR data, the mutants of 1G2 that were compromised in their ability to bind to gB had higher $\mathrm{EC}_{50}$ values compared with WT 1G2 (Table 2). It is likely that avidity effects, due to the bivalent nature of antibody binding, account for the moderate increase in $\mathrm{EC}_{50} \mathrm{~s}$ (two to sixfold) observed for most of the mutations compared with the more pronounced effect observed on $\mathrm{gB} / 1 \mathrm{G} 2$ binding affinities. However, the double mutant Tyr103Ala/Phe104Ala, which binds poorly to $\Delta \mathrm{NgB}$ glyco, had an $\mathrm{EC}_{50}$ value nearly 68 -fold higher than WT $1 \mathrm{G} 2$ $\left(6.79 \mu \mathrm{g} \mathrm{ml}^{-1}\right.$ and $0.1 \mu \mathrm{g} \mathrm{ml}^{-1}$, respectively). In contrast, other mutations in HCDR1 (Arg31Ser and Ser32Ala), HCDR3 (Tyr109Ala) and LCDR2 (Tyr50Ala and Arg51Ala) did not alter the neutralizing capacity of 1G2 significantly. Overall, the results of the mutagenesis studies are in agreement with the structural data.

Functional characterization of 1G2. To further functionally characterize $1 \mathrm{G} 2$, we tested its breadth of neutralization on a number of HCMV clinical and laboratory strains (Table 3). 1G2 was able to effectively neutralize entry of all 10 strains tested in both epithelial and fibroblast cells in a complement-independent manner with average $\mathrm{EC}_{50}$ values of $0.246 \mu \mathrm{g} \mathrm{ml}^{-1}(1.64 \mathrm{nM})$ or $0.311 \mu \mathrm{g} \mathrm{ml}^{-1}(2 \mathrm{nM})$, respectively. We also assessed potential differences between 1G2 IgG and Fab by investigating their neutralization properties against the HCMV clinical strain VR1814. As previously reported ${ }^{36}$, the Fab of $1 G 2$ was as effective as the whole IgG in neutralizing the virus in epithelial cells, with an $\mathrm{EC}_{50}$ value of $0.15 \mu \mathrm{g} \mathrm{ml}^{-1}(3 \mathrm{nM})$ compared with $0.21 \mu \mathrm{g} \mathrm{ml}^{-1}(1.42 \mathrm{nM})$ for the IgG (Table 3).

To gain insights into the molecular basis for the broad neutralizing properties of $1 \mathrm{G} 2$, we aligned the sequences of several clinical and laboratory strains of gB (Supplementary Fig. 5) and polymorphisms in the structured parts of the ectodomain were mapped to the surface of HCMV gB (coloured in cyan in Fig. 6a). It is apparent that unlike the AD-1 epitope, no known polymorphisms map to the epitopes of $1 \mathrm{G} 2 \mathrm{mAb}$ in $\mathrm{AD}-5$ or SM5 $\mathrm{mAb}$ in $\mathrm{AD}-4$, further underscoring the broad neutralizing capacity of these mAbs. One polymorphism at position 287 (Ala or Thr) falls in the proximity of the 1G2 epitope, but in the structure, Ala287 is solvent exposed and does not form any contacts with the Fab (Fig. 4b), consistent with the similar neutralization potency of $1 \mathrm{G} 2$ on both variants (Table 3 ).

Finally, we tested whether 1G2 could bind to gB in the context of the membrane-anchored full-length protein. BHK cells were electroporated with RNA encoding full-length WT HCMV gB and the cell surface was stained with 1G2 or ITC88 (an antibody targeting the $\mathrm{gB} \mathrm{AD}-2$ epitope previously shown to bind cell surface-expressed and virion $\mathrm{gB}^{43}$ ). Both antibodies recognized gB expressed on the cell surface to a similar extent. Next, purified VR1814 virions were immunoprecipitated with 1G2 to determine if the epitope is accessible on the viral surface (Fig. 5b). ITC88 was used as a positive control. The 1G2 antibody immunoprecipitated $\mathrm{gB}$ from virions, confirming that the epitope is accessible on the viral surface. Together these results suggest that $1 \mathrm{G} 2$ binds to a functionally important site that is exposed and accessible to the antibody on both the cell and viral surfaces.

Mapping known neutralizing epitopes on HCMV gB. Neutralizing epitopes in HCMV and HSV gB have been identified using peptide mapping, escape mutants and more recently by site-directed mutagenesis ${ }^{11,32,33,35,36,38}$. While antibodies to HCMV gB AD-1, AD-4 and AD-5 recognize conformational epitopes, $\mathrm{AD}-2$ antibodies recognize linear sequences in the flexible $\mathrm{N}$ terminus of $\mathrm{gB}^{32}$. In a comparison of the known sites mapped on the respective postfusion gB structures (Fig. 6a,b), the footprint of $1 \mathrm{G} 2$ Fab overlaps with residues in HSV gB known to be critical for binding of several neutralizing antibodies (for example, H126, H1375, B4 and H1435) ${ }^{11}$. Likewise, the epitope for SM5, recently characterized in a crystal structure with an isolated AD-4 domain ${ }^{37}$, overlaps with that for H1838 in HSV gB. The known epitope in $\mathrm{AD}-1$ is in a groove on the top of the crown and close to the trimer axis ${ }^{32}$. A point mutation conferring resistance to the $\mathrm{B} 2$ and $\mathrm{B} 5$ antibodies in $\mathrm{HSV} \mathrm{gB}$ falls in a similar location. By contrast, an additional neutralizing epitope in HSV gB targeted by the SS10 antibody and mapped to the outer surface of the crown ${ }^{31}$ (Fig. 6b) has not been described for HCMV gB.

Depletion of neutralizing antibodies in human sera by $\mathrm{gB}$. To assess the contribution of antibodies targeting different HCMV envelope glycoproteins to neutralization, sera from five seropositive subjects were incubated with purified Pentamer, $\mathrm{gH} / \mathrm{gL}^{44}$, or postfusion $\mathrm{gB}$ (gB-698glyco) and the corresponding antibody-depleted sera were evaluated for neutralization of epithelial cell and fibroblast infection (Table 4). The neutralization experiments were performed in the presence of complement because virion $\mathrm{gB}$ raises for the most part complement-dependent neutralizing responses. The specificity and efficiency of depletion were confirmed with an ELISA 
a
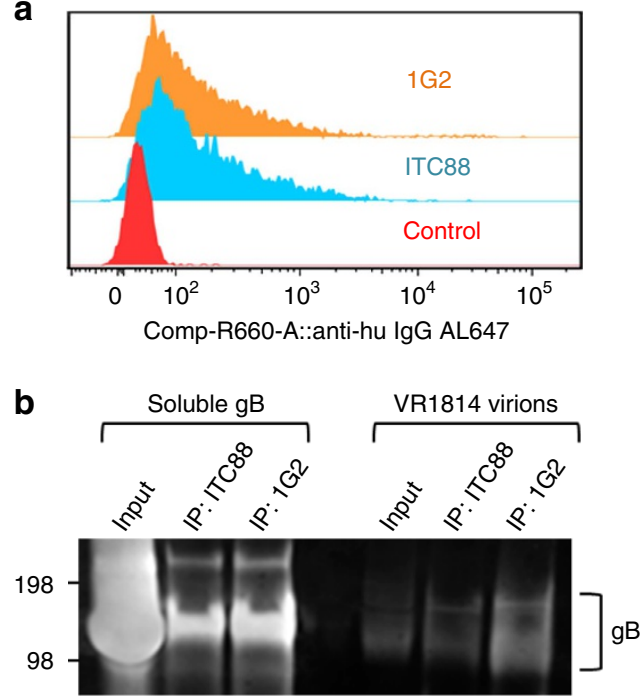

Figure 5 | Binding of 1G2 to full-length gB on cell and viral surface. (a) FACS analysis of BHK cells electroporated with RNA encoding full-length gB and stained with monoclonal antibodies 1G2 and ITC88 $24 \mathrm{~h}$ post electroporation. The $\mathrm{gB}$ staining histograms were gated on viable, dsRNA + cells. (b) Western blot analysis of purified VR1814 virions immunoprecipitated with $1 \mathrm{G} 2$ or ITC88. Samples were resolved on SDS-polyacrylamide gel electrophoresis and detected with ITC88 mAb. The left panel shows immunoprecipitation of soluble gB-698glyco by both antibodies as control.

(Supplementary Fig. 6). As previously reported, HCMV Pentamer was confirmed to be the main target of antibodies inhibiting HCMV infection of epithelial cells ${ }^{45}$. Notably, while immunization with $\mathrm{gH} / \mathrm{gL}$ is capable of raising antibodies that inhibit HCMV infection in both fibroblasts and epithelial cells ${ }^{44}$, no reduction in sera neutralization of epithelial cell infection was noted on depletion with $\mathrm{gH} / \mathrm{gL}$, reflecting the superior potency of antibodies specific for the pentamer in neutralizing epithelial cell infection $^{34,46}$. Similarly, no observable difference in neutralizing titer is noted following depletion with purified postfusion $\mathrm{gB}$ when assayed on epithelial cells (Table 4), despite the exposure of the 1G2 epitope on virion $\mathrm{gB}$ (this study). By contrast, purified gB-698glyco was able to deplete a large fraction of the complement-dependent neutralizing activity of fibroblast infection by HCMV (Table 4). For three of the five subjects, depletion with $\mathrm{gB}$ brought neutralizing titres to below the detection limit, while the other two were reduced to nearly a third of the original titres. Interestingly, neither Pentamer nor $\mathrm{gH} / \mathrm{gL}$ complexes showed significant neutralization depletion for fibroblast infection. Therefore, most of the epitopes recognized by gB-specific antibodies contained in sera from seropositive individuals and capable of neutralizing fibroblast infection in presence of complement are exposed on the postfusion $\mathrm{gB}$ molecule.

\section{Discussion}

HCMV is a medically relevant human pathogen and a target for therapeutic intervention. HCMV gB is a key target for antibody development and remains a likely component of any future HCMV vaccine ${ }^{17}$. Here we have reported the crystal structure of an engineered homogeneous postfusion HCMV gB ectodomain trimer bound to a Fab derived from the neutralizing human monoclonal antibody $1 \mathrm{G} 2$. This represents the first atomic structure of an HCMV envelope glycoprotein.
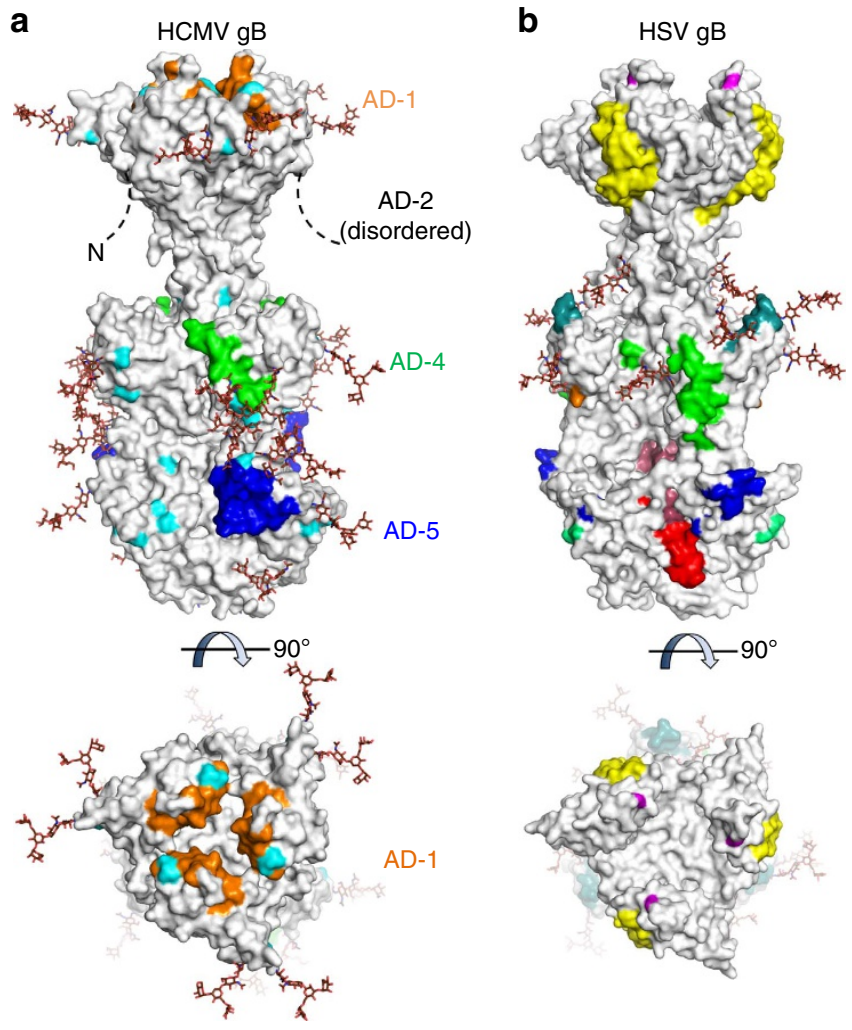

Figure 6 | Neutralizing epitopes in HCMV gB. (a) Surface representation of $\mathrm{HCMV} g B$ in its postfusion conformation with known neutralizing epitopes in AD-1 (domain IV) in orange, AD-4 (domain II) in green and AD-5 (domain I) in blue. These epitopes are composed of residues Tyr280-Phe300 in AD-5 (mAb 1G2, this report), residues Ala360-Tyr364 and Ser377-Asp385 in AD-4 (mAb SM5 (ref. 37)) and residues Pro571-Val580 and His606-Phe619 in AD-1 (mAbs ITC48, ITC52, ITC63B and ITC $63 C^{32}$ ). The epitopes in AD-4 and AD-5 have been defined based on crystal structures while the epitope in AD-1 was identified through peptide mapping. Polymorphisms among clinical and laboratory strains of HCMV are shown in cyan. (b) Surface representation of HSV gB (PDB ID: 2GUM) with neutralizing epitopes defined through peptide mapping and single amino acid resistant mutant studies ${ }^{11,31,64}$. Residues recognized by mAbs H233 (Ala315 and Arg328), H126 (Tyr303), H1375 (Arg304), B4 (Glu305) and H1435 (His308) are shown in blue; mAb SS55 (Asp199 and Ala203) in light green; mAb H1781 (Pro454-Ser473) in teal; mAb H1838 (Ala390-Gly410) in green; mAb C226 (Asp419) in orange; mAb SS10 (Tyr640-Phe670) in yellow; mAbs B2 and B5 (Gly594) in magenta; mAb SS106 (Ser697-Ala725) in pink; mAb SS144 (Arg715-Ala725) in red. Simple (oligomannose) glycans shown as brown sticks were modelled into glycosylation sites in the primary sequence of HCMV and HSV gB using the Glyprot server.

1G2, SM10 and 2C2 antibodies were isolated from B-cell repertoire analysis from three seropositive subjects and mutagenesis studies suggested that they bind to the AD-5 site in $\mathrm{gB}^{33}$. Our EM analysis and the crystal structure of the complex confirmed the location of the epitope and revealed the atomic details of this interaction. $1 \mathrm{G} 2$ contacts gB mainly with the $\mathrm{V}_{\mathrm{H}}$ domain burying a large hydrophobic surface on $\mathrm{gB}$. The almost exclusive use of the heavy chain for binding is unusual ${ }^{47}$ and reminiscent of the influenza virus broadly neutralizing antibody CR6261 that, similar to $1 \mathrm{G} 2$, binds to a hydrophobic surface on the HA molecule ${ }^{48}$. The interaction is characterized by 1 G2 HCDR3, rich in aromatic amino acids, and HCDR1 protruding into a hydrophobic cleft within AD-5. Our mutagenesis data confirmed 
Table 4 | Neutralization activity in human sera after antibody depletion with $\mathrm{gB}, \mathrm{gH} / \mathrm{gL}$ and pentamer.

\begin{tabular}{|c|c|c|c|c|c|c|}
\hline \multirow[t]{2}{*}{ Cell type } & \multirow[t]{2}{*}{ Depleting protein } & \multicolumn{5}{|c|}{ Human serum } \\
\hline & & 1 & 2 & 3 & 4 & 5 \\
\hline \multirow[t]{4}{*}{ Fibroblasts } & None & 2,254 & 4,093 & 2,442 & 4,488 & 3,328 \\
\hline & gB-698glyco & 100 & 100 & 768 & 1,266 & 100 \\
\hline & $\mathrm{gH} / \mathrm{gL}$ & 1,598 & 3,689 & 5,738 & 3,523 & 3,921 \\
\hline & Pentamer & 1,607 & 1,363 & 2,240 & 3,761 & 4,569 \\
\hline \multirow[t]{4}{*}{ Epithelial cells } & None & 4,126 & 11,407 & $\geq 12,800$ & $\geq 12,800$ & 4,867 \\
\hline & gB-698glyco & 3,972 & $\geq 12,800$ & $\geq 12,800$ & $\geq 12,800$ & 5,086 \\
\hline & $\mathrm{gH} / \mathrm{gL}$ & 4,561 & 12,422 & $\geq 12,800$ & $\geq 12,800$ & 5,636 \\
\hline & Pentamer & 720 & 1,104 & 1,408 & 1,708 & 1,464 \\
\hline
\end{tabular}

gB, glycoprotein B; HCMV, human cytomegalovirus.

Sera from five convalescent seropositive human subjects were assessed for neutralization potential on fibroblasts (MRC-5 cells) following infection with HCMV Towne strain, or epithelial cells (ARPE-19 cells) following infection with HCMV TB40/E strain. The respective strains were chosen to achieve optimal infection of the epithelial and fibroblast cells. All neutralization assays were carried out in the presence of complement. Depletion with $\mathrm{gB}-698 \mathrm{glyco}, \mathrm{gH} / \mathrm{gL}$ or Pentamer was carried out by incubating the sera with the proteins for $1 \mathrm{~h}$ before the neutralization assay.

the critical role of several of these aromatic residues for tight binding to $\mathrm{gB}$ and virus neutralization.

It is interesting to note that among all the isolated neutralizing mAbs targeting $\mathrm{gB} \mathrm{AD}-5,1 \mathrm{G} 2$ has the shortest $\mathrm{HCDR} 3$ (12 residues versus 17 for SM10 and 24 for 2C2) (refs 33,36). In addition, sequence comparisons reveal that not all of the $\mathrm{AD}-5$ antibodies have hydrophobic CDRs, suggesting that presence of hydrophobic CDRs is not an absolute requirement for binding to the AD-5 site ${ }^{33}$. Of note, 1G2, SM10 and 2C2 belong to different germlines suggesting that there is no specific genetic requirement to elicit antibodies binding to this epitope.

The structure of postfusion $\mathrm{gB}$ reveals the location and structure of epitopes recognized by other neutralizing human mAbs, and our structural analysis demonstrates that most of these epitopes are common to both HCMV and HSV gBs. This observation suggests a conserved functional role of these sites across herpesvirus gBs. A recent report described the structure of an HCMV AD-4 only molecule bound to the SM5 Fab ${ }^{37}$. The postfusion $\mathrm{gB}$ trimer structure confirms that this epitope is exposed and has a very similar structure in the context of the entire $\mathrm{gB}$ ectodomain. $\mathrm{AD}-1$, on the crown, was identified using peptides. Interestingly, when mapped on the postfusion $\mathrm{gB}$ structure, this site seems to point towards the three-fold axis. Therefore the epitope is likely not accessible to antibodies in the postfusion conformation. This observation points to a potential limitation of the postfusion $\mathrm{gB}$ molecule as a subunit vaccine antigen in eliciting neutralizing antibodies binding to this site in $\mathrm{AD}-1$.

Mouse immunization studies with different postfusion HCMV gB trimer preparations were shown to raise almost exclusively complement-dependent neutralizing antibodies ${ }^{44,49}$. However, the structure of postfusion $\mathrm{gB}$ reveals that sites capable of binding complement-independent neutralizing antibodies are present in this form of the $\mathrm{gB}$ molecule suggesting that other epitopes are dominant. Consistent with this hypothesis, the structure also showed that both $\mathrm{AD}-4$ and $\mathrm{AD}-5$ are surrounded by glycosylation sites, which may limit epitope exposure, and the neutralizing site in AD-2 may be only partially accessible to antibody binding. The situation seems similar to the case of natural infection where only a small percentage of the antibodies targeting $\mathrm{gB}$ are neutralizing ${ }^{33,50}$. We speculate that, similar to other viruses ${ }^{51}$, the majority of the fusion proteins on the viral surface are in the postfusion conformation providing an escape mechanism. Therefore, antigen design strategies further modifying the postfusion $\mathrm{gB}$ trimer to focus the immune response towards neutralizing sites (for example, AD-4 and $\mathrm{AD}-5)$ and dampening the non-neutralizing sites may result in an improved vaccine antigen. For instance, selective mutation of glycosylation sites surrounding the 1G2 epitope may increase its exposure and elicit more neutralizing antibodies binding to this epitope.

In summary, the structure of the postfusion $\mathrm{HCMV} \mathrm{gB}$ trimer reveals structural and antigenic similarities with other herpesvirus gB likely due to the conserved functional role of these molecules. The structural and antigenic analyses show that the postfusion trimer presents complement-independent neutralizing sites. Thus modifications on the $\mathrm{gB}$ trimer surface that decrease exposure of non-neutralizing sites may result in an improved vaccine. Conversely, potent HCMV neutralizing antibodies, like 1G2, are attractive candidates for treatment of HCMV infection. Individual $\mathrm{mAbs}$, or combinations of neutralizing antibodies targeting the different domains within $\mathrm{gB}$ and/or the HCMV $\mathrm{gH} / \mathrm{gL}$-based complexes may provide a suitable strategy.

\section{Methods}

Generation of HCMV glycoprotein and Fab constructs. Merlin strain HCMV gB codon-optimized for expression in mammalian cells, with an optimal Kozak sequence immediately 5 ' to the gene, was synthesized by GeneArt (Regensburg, Germany) and subcloned into the SalI and XbaI restriction sites of the eukaryotic expression vector pCMVKm2 (ref. 52). The gB gene was truncated at amino acid 698 to remove the transmembrane domain and cytoplasmic tail and fused to a 6-histidine tag for purification purposes. Point mutations of Ile156His, His157Arg Trp240Arg, Cys246Ser, Arg457Ser, Arg460Ser, were introduced by site-directed mutagenesis using a QuikChange II XL Site Directed Mutagenesis Kit (Agilent) resulting in gB-698. Addition of the mutations Trp240Asn and Tyr242Thr to gB-698 or deletion of residues $26-87$ together with the Trp240Asn and Tyr242Thr mutations in gB-698 generated gB-698glyco and $\triangle \mathrm{NgB}$-glyco, respectively. Recombinant $1 \mathrm{G} 2 \mathrm{IgG}$ and Fab were cloned based on the published heavy and light chain sequences ${ }^{3}$. The recombinant IgG was cloned into an IgG1 backbone. The Fab was expressed with a cleavable C-terminal Strep tag II on the heavy chain.

Expression and purification of $\mathbf{g B}, \mathbf{1 G 2}$ and $\mathbf{g B} / \mathbf{1 G 2}$ complexes. 1G2 Fab was transiently expressed in 293EBNA cells (ATCC, Manassas, VA), gB-698glyco was stably expressed in $\mathrm{CHO} \mathrm{lec}^{-}$cells whereas $\triangle \mathrm{NgB}$-glyco was transiently expressed in HEK293S GnTI ${ }^{-}$cells (ATCC, Manassas, VA). For purification of all the gB constructs, expression media were concentrated 10 -fold and buffer exchanged into $25 \mathrm{mM}$ Tris pH 7.5, $300 \mathrm{mM} \mathrm{NaCl}$ using a tangential flow filtration system (Millipore). Imidazole to a final concentration of $10 \mathrm{mM}$ was added to sample before loading onto a Ni-NTA Superflow cartridge (Qiagen) using the Akta Explorer 100 Air system (GE Lifesciences). His-tagged gB was eluted using a continuous gradient of $10-500 \mathrm{mM}$ imidazole with the protein typically eluting around $200 \mathrm{mM}$. Fractions dialysed into $25 \mathrm{mM}$ Tris pH 7.5, $150 \mathrm{mM} \mathrm{NaCl}$ were further purified using size exclusion chromatography on a Superose 6 column (GE Lifesciences). The proteins were concentrated to final concentrations of $7 \mathrm{mg} \mathrm{ml}^{-1}$ for gB-698glyco and $3.5 \mathrm{mg} \mathrm{ml}^{-1}$ for $\Delta \mathrm{NgB}$-glyco. Strep-tagged $1 \mathrm{G} 2$ Fab was affinity purified using a Strep-Tactin Superflow Plus column (Qiagen) and concentrated to $5.3 \mathrm{mg} \mathrm{ml}^{-1}$

For deglycosylation experiments, Ni-purified $\mathrm{gB}$ ectodomain constructs were incubated at room temperature (RT) for $20 \mathrm{~min}$ with Endo Hf (New England Biolabs) according to manufacturer's instructions. The protein was subsequently purified by SEC as described above and concentrated to $3.7 \mathrm{mg} \mathrm{ml}^{-1}$ (gB-698glyco) or $1.74 \mathrm{mg} \mathrm{ml}^{-1}$ ( $\Delta \mathrm{NgB}$-glyco). 
EM for $\mathbf{g B}$ and $\mathbf{g B} / \mathbf{1 G 2}$ Fab complexes. For EM analysis, gB-698glyco was incubated with 1G2 Fab at a 1:1.5 molar ratio on ice for $1 \mathrm{~h}$ and resolved over a Superose 6 PC 3.2/30 column connected to an Akta Micro system (GE Lifesciences). For visualization, $5 \mu \mathrm{l}$ of sample was incubated on a freshly discharged continuous carbon 400-mesh copper grid (Electron Microscopy Sciences) for $30 \mathrm{sec}$. Following incubation, the grid was moved through $5 \times 75 \mu \mathrm{l}$ droplets of a $2 \%(\mathrm{w} / \mathrm{v})$ uranyl formate solution (SPI Supplies). Excess stain solution was blotted away and the grid was air-dried. EM images were collected on a Tecnai-12 Spirit (FEI) equipped with a LaB6 filament operated at $120 \mathrm{keV}$ under low dose conditions using a $4 \mathrm{k} \times 4 \mathrm{k} C \mathrm{CD}$ camera (Gatan Inc.) at a nominal magnification of $\times 49,000$ ( $2.15 \AA$ per pixel). Particles were isolated from individual micrographs using the e2boxer.py procedures embedded into EMAN2 (ref. 53) and subjected to iterative two-dimensional reference-free image analysis using multivariate statistical analysis followed by multi-reference alignment in IMAGIC5 (ref. 54). To obtain a gB/1G2 three-dimensional reconstruction, $\sim 10,000$ particles were refined against an initial model of HSV gB (PDB ID 2GUM) low pass filtered to a resolution of $60 \AA$ using the EMAN2/Sparx software ${ }^{53,55}$ and fitted in Chimera ${ }^{56}$.

Crystallization of $\mathbf{g B / 1 G 2}$ Fab complexes. All crystallization trials were carried out at $20^{\circ} \mathrm{C}$. In initial crystallization experiments, gB-698glyco was mixed with 1G2 Fab at a molar ratio of 1:1.2, and 1,000:1 w/w of Subtilisin E (Hampton Research) and directly screened using sparse matrix crystallization screens. Crystallization screens were set-up using a Mosquito Crystal liquid handler (TTP Labtech) in 96-well hanging drop format and imaged using a RockImager robot (Formulatrix Inc). Initial crystals appeared overnight in a drop containing $0.1 \mu \mathrm{l}$ protein and $0.1 \mu \mathrm{l}$ reservoir solution of $0.1 \mathrm{M}$ MMT buffer pH 9.0, 25\% PEG-1500 (Wizard IV crystallization screen, Emerald Biosystems). Final crystals were obtained in 15-well screw cap hanging drop plates (Qiagen Inc) by microseeding into $0.1 \mathrm{M}$ MMT pH 8.4, 12\% PEG-1500, 5\% glycerol after overnight equilibration. Screw caps with crystals were transferred to a well containing 0.1 M MMT pH 8.4, 25\% PEG1500, 20\% glycerol for dehydration overnight, and crystals were directly flash-frozen in liquid nitrogen. Crystals of deglycosylated $\Delta \mathrm{NgB}$-glyco/1G2 Fab were obtained under similar conditions with no protease treatment, and used as seeds for obtaining single crystals in a drop containing a 1:1 volume ratio of protein complex to $40 \mathrm{mM}$ MMT pH 9, $8 \%$ PEG8K and $0.1 \mathrm{M} \mathrm{NaCl}$ and $20 \%$ glycerol. Crystals were directly flash frozen in liquid nitrogen for data collection.

\section{Structure determination of $\Delta \mathbf{N g B}-$ glyco/1G2 Fab complex. Data collection was} carried out with a $50 \%$ attenuated beam at the PXII beamline at the Swiss Light Source (SLS, Switzerland). Data were scaled and processed using XDS autoPROC (Globalphasing Inc) to $3.6 \AA$ resolution for subsequent analysis and structure determination. Matthew's coefficient analysis within the CCP4 suite ${ }^{57}$ predicted a single monomer per asymmetric unit with $67 \%$ solvent content. Molecular replacement (MR) was carried out using Phaser (CCP4 suite) ${ }^{58}$ with the HSV gB ectodomain structure as search model (PDB ID: 2GUM). A single solution with gB along the three-fold axis was obtained with an $R_{\text {work }} / R_{\text {free }}$ of $0.55 / 0.56$. Initial electron density was clearest for the central helix while densities in structural domains I and IV were weakest. The initial $\mathrm{gB}$ model was further improved by iterative cycles of model building in Coot, model refinement using Sculp $\mathrm{t}^{59}$ within Phenix ${ }^{60}$ and electron density modification with DM (CCP4 suite). Cycles of model refinement with Phenix ${ }^{61}$ using only TLS parameters, $x y z$ coordinates options combined with kicked electron density maps and model building in $\mathrm{Coot}^{62}$ were performed until the $R_{\text {work }}$ and $R_{\text {free }}$ reached 0.26 and 0.31 , respectively.

For placing the Fab, a carbon alpha trace for HCDR3 was first built into a well-defined electron density at the complex interface. Then the $V_{H} V_{L}$ chains of the Fab could be located by molecular replacement with Phaser. No clear solution could be identified for the $\mathrm{C}_{\mathrm{H}}$ and $\mathrm{C}_{\mathrm{L}}$ domains of the Fab. After several cycles of model building and refinement the final $R_{\text {work }}$ and $R_{\text {free }}$ values decreased to 0.21 and 0.26 , respectively (Table 1 ). While the crystal packing allows enough space for the constant region of the Fab, the electron density is mostly discontinuous suggesting that this region is flexibly linked to the variable region and therefore not visible because it likely adopts different orientations in the crystals. Structure validation was carried out using Molprobity ${ }^{63}$. The coordinates for the structure have been deposited with the RCSB Protein Data Bank under PDB ID 5C6T.

SPR experiments for $\mathbf{G B / 1 G 2}$ binding. SPR single cycle kinetic experiments were carried out using a Biacore T100 instrument. Two adjacent channels on a CM5 sensor chip were immobilized with IgG binder using the Human Antibody Capture Kit (GE Healthcare BR100839) according to manufacturer's recommendations. HBS-EP buffer (GE healthcare BR100669) was used as diluent for both ligand and analyte samples. Ligand 1G2 IgG was captured on one channel (leaving the other channel as reference). For the 1G2 mutants, cell culture supernatant expressing the various mutants was flowed over the human IgG binder for the capture step. Capture levels were around 200 resonance units (RUs) for analysis of the $\Delta \mathrm{NgB}$ glyco mutants and roughly 40 RUs for analysis of the 1G2 mutants. For both experiments, $\Delta \mathrm{NgB}$-glyco at $0,1.56,3.125,6.25,12.5$ and $25 \mathrm{nM}$ were injected over the two channels at $50 \mu \mathrm{lmin}{ }^{-1}$ for $120 \mathrm{~s}$ followed by $600 \mathrm{~s}$ of dissociation time. The single cycle kinetic curves were fitted with 1:1 binding stoichiometry for $k_{\mathrm{a}}, k_{\mathrm{d}}$ and $K_{\mathrm{D}}$
Determination of concentration of $\mathbf{1 G 2}$ mutants using SPR. To determine the concentration of $1 \mathrm{G} 2$ and its point mutants in cell culture supernatant, an SPR-based concentration analysis method was used. Briefly, a calibration curve was set-up using purified $1 \mathrm{G} 2$ at concentrations ranging from $10 \mu \mathrm{g} \mathrm{ml}^{-1}$ to $3.2 \mathrm{ng} \mathrm{ml}^{-1}$ in fivefold dilutions by capturing the antibody on a human IgG binder chip described above. A 1:100 dilution of cell culture supernatant from 293 Expi cells (Invitrogen Inc.) transfected with WT or point mutants of 1G2 was then flowed over the chip and the resulting capture level recorded. The final concentration of the antibodies was determined by the concentration analysis using calibration program within the Biacore T200 evaluation software.

\section{Surface staining of full-length gB by flow cytometry. BHK cells were} electroporated with $0.1 \mu \mathrm{g}$ of FL-gB RNA along with $4.2 \mu \mathrm{g}$ of mouse thymus RNA. Following overnight culture (16-18 h) the cells were detached using Trypsin-EDTA (Invitrogen Inc.). Cells were initially stained for viability using a LIVE/DEAD Fixable Yellow Dead Cell Stain Kit (Life Technologies) and were then stained for surface expression of gB with either ITC88 (1 $\mu \mathrm{g}$ per sample), 1G2 (1 $\mu \mathrm{g}$ per sample) or none (as control), followed by goat anti-human IgG $(\mathrm{H}+\mathrm{L})$ Alexa Fluor 647 ( $0.25 \mu \mathrm{g}$ per sample; Cat no. A-21445, Life Technologies). Cells were fixed and permeabilized, and then stained with an anti-dsRNA mAb $(0.25 \mu \mathrm{g}$ per sample; clone J2, Cat no. 10010500, English and Scientific Consulting Bt) that had been conjugated using a Zenon R-Phycoerythrin Mouse IgG2a Labeling Kit (Life Technologies). All samples were analysed by flow cytometry on an LSR II instrument (BD Biosciences).

Virus neutralization assay with 1G2 mutants. 1G2 WT or various point mutants were expressed in 293Expi cells in a 24-well format. Supernatant from all samples were verified for antibody expression 4 days post-transfection on a Coomassiestained SDS-polyacrylamide gel electrophoresis gel. Supernatant samples were serially diluted in twofold steps (two replicates per dilution), mixed with an equal volume of HCMV virus (concentration of 200-250 infected cells/counting field) in media $+10 \%$ guinea pig complement (Cedarlane Labs, Burlington, NC, USA) and incubated for $2 \mathrm{~h}$ at $37^{\circ} \mathrm{C} / 5 \% \mathrm{CO}_{2}$. These serum/virus samples were added to ARPE-19 cells prepared in 96-well half-area cell culture plates (Corning Inc., Corning, NY, USA) plated at a density of $6.25 \times 10^{4}$ per well 1 day before infection. The infected monolayers were incubated for $48 \mathrm{~h}( \pm 8 \mathrm{~h})$ at $37^{\circ} \mathrm{C} / 5 \% \mathrm{CO}_{2}$, fixed with $10 \%$ buffered formalin (EMD Chemicals Inc., Gibbstown, NJ, USA) for $1 \mathrm{~h}$, washed three times with wash buffer (PBS/0.05\% Tween-20) and blocked with $\mathrm{PBS} / 2.5 \% \mathrm{FBS}, 0.5 \%$ saponin and $0.1 \%$ sodium azide for $1 \mathrm{~h}$ at RT. Following three washes, plates were tapped dry and incubated in a $25^{\circ} \mathrm{C}$ humid incubator for $1 \mathrm{~h}$. The plates were then incubated for $1 \mathrm{~h}$ at RT with anti-HCMV IE1 antibody derived from hybridoma L14. Plates were washed three times and incubated for $1 \mathrm{~h}$ with anti-mouse IgG conjugated with Alexa Fluor 488, and then washed three times with PBS/0.05\% Tween-20. The fluorescent cells were counted using an Immunospot S5 UV Analyzer (Cellular Technology Limited, Shaker Heights, OH, USA), and the $50 \%$ neutralization titer, defined as the reciprocal of the serum dilution yielding $50 \%$ reduction in the infected cell count relative to control, was calculated by linear regression interpolation between the two dilutions with wells yielding average infected cell counts above and below the $50 \%$ value.

Human sera depletion assay. Sera from five human subjects seropositive for CMV antibodies were obtained from SeraCare Life Sciences (Milford, MA, USA). Serum samples were heat inactivated at $56^{\circ} \mathrm{C}$ for $30 \mathrm{~min}$ and subsequently incubated for $1 \mathrm{~h}$ at RT with and without purified soluble gB-698glyco, gH/gL or pentamer at a concentration of $40 \mu \mathrm{g} \mathrm{ml}^{-1}$. These depleted and non-depleted samples were then assayed in the neutralization assay as described above. To determine the specificity and efficiency of depletion, sera incubated with soluble gB-698glyco, gH/gL or Pentamer as described above were assayed in an ELISA with the respective proteins coated onto plates. Sera incubated with an unrelated protein ('mock') were used as a control.

Neutralization assay with multiple strains of HCMV. HCMV virus from various strains was pre-incubated with 10-fold serial dilutions of 1G2 antibody (from 100 to $0.0001 \mu \mathrm{g} \mathrm{ml}^{-1}$ ) for $1 \mathrm{~h}$ before infection. ARPE-19 or NHDF cells (ATCC, Manassas VA) seeded at 10,000 per well were infected in a 96-well plate with the virus antibody complex for $3 \mathrm{~h}$ in a serum free medium, followed by a wash with complete medium. Fourty-eight hours post infection, cells were fixed with $4 \%$ paraformaldehyde and permeabilized with $0.1 \%$ Triton-X100. Mouse anti-IE antibody 6F8.2 (Chemicon) was diluted 1:2,000 and incubated on the cells for $1 \mathrm{~h}$ at RT following which cells were washed three times in $1 \times$ PBS. Anti-mouse AF594 $(1: 3,000)$ and DAPI $(1: 5,000)$ were diluted in $1 \times$ PBS and incubated on cells for $1 \mathrm{~h}$ at RT. Cells were washed three times in 1PBS and the number of IE1/2 positive cells were counted using a Cellomics Arrayscan.

gB immunoprecipitation. Soluble gB ectodomain or an equal amount of cushion-purified VR1814 virions (500 $\mu \mathrm{g}$ per reaction) were lysed in 1\% NP40 and incubated with $10 \mu \mathrm{g}$ of $1 \mathrm{G} 2$ or ITC88 antibodies for $16 \mathrm{~h}$ at $4{ }^{\circ} \mathrm{C}$. Complexes were immunoprecipitated using Dynabeads Protein A kit from Life technologies 
(Cat. no. 10006D) according to the manufacturer's protocol. The complexes were washed four times in lysis buffer. Samples were boiled for $5 \mathrm{~min}$ at $96^{\circ} \mathrm{C}$ before immunoblotting with ITC88. The uncropped western blot image is shown in Supplementary Fig. 7.

\section{References}

1. Kenneson, A. \& Cannon, M. J. Review and meta-analysis of the epidemiology of congenital cytomegalovirus (CMV) infection. Rev. Med. Virol. 17, 253-276 (2007).

2. Kotton, C. N. Management of cytomegalovirus infection in solid organ transplantation. Nat. Rev. Nephrol. 6, 711-721 (2010).

3. Snydman, D. R. Cytomegalovirus immunoglobulins in the prevention and treatment of cytomegalovirus disease. Rev. Infect. Dis. 12(Suppl 7): S839-S848 (1990).

4. Arvin, A. M., Fast, P., Myers, M., Plotkin, S. \& Rabinovich, R. National Vaccine Advisory C. Vaccine development to prevent cytomegalovirus disease: report from the National Vaccine Advisory Committee. Clin. Infect. Dis. 39, 233-239 (2004).

5. Stratton, K. R., Durch, J. S. \& Lawrence, R. S. Vaccines for the 21st Century-A tool for decision making (National Academy Press, 2001).

6. Connolly, S. A., Jackson, J. O., Jardetzky, T. S. \& Longnecker, R. Fusing structure and function: a structural view of the herpesvirus entry machinery. Nat. Rev. Microbiol. 9, 369-381 (2011).

7. Vanarsdall, A. L., Chase, M. C. \& Johnson, D. C. Human cytomegalovirus glycoprotein $\mathrm{gO}$ complexes with $\mathrm{gH} / \mathrm{gL}$, promoting interference with viral entry into human fibroblasts but not entry into epithelial cells. J. Virol. 85, 11638-11645 (2011).

8. Vanarsdall, A. L. \& Johnson, D. C. Human cytomegalovirus entry into cells. Curr. Opin. Virol. 2, 37-42 (2012).

9. Wang, D. \& Shenk, T. Human cytomegalovirus virion protein complex required for epithelial and endothelial cell tropism. Proc. Natl Acad. Sci. USA. 102, 18153-18158 (2005).

10. Zhou, M., Yu, Q., Wechsler, A. \& Ryckman, B. J. Comparative analysis of $\mathrm{gO}$ isoforms reveals that strains of human cytomegalovirus differ in the ratio of $\mathrm{gH} / \mathrm{gL} / \mathrm{gO}$ and $\mathrm{gH} / \mathrm{gL} / \mathrm{UL} 128-131$ in the virion envelope. J. Virol. 87, 9680-9690 (2013).

11. Heldwein, E. E. et al. Crystal structure of glycoprotein B from herpes simplex virus 1. Science 313, 217-220 (2006).

12. Wille, P. T., Wisner, T. W., Ryckman, B. \& Johnson, D. C. Human cytomegalovirus (HCMV) glycoprotein $\mathrm{gB}$ promotes virus entry in trans acting as the viral fusion protein rather than as a receptor-binding protein. mBio 4, e00332-00313 (2013).

13. Atanasiu, D. et al. Bimolecular complementation defines functional regions of Herpes simplex virus gB that are involved with $\mathrm{gH} / \mathrm{gL}$ as a necessary step leading to cell fusion. J. Virol. 84, 3825-3834 (2010).

14. Avitabile, E., Forghieri, C. \& Campadelli-Fiume, G. Cross talk among the glycoproteins involved in herpes simplex virus entry and fusion: the interaction between $\mathrm{gB}$ and $\mathrm{gH} / \mathrm{gL}$ does not necessarily require $\mathrm{gD}$. J. Virol. 83, 10752-10760 (2009).

15. Vanarsdall, A. L., Ryckman, B. J., Chase, M. C. \& Johnson, D. C. Human cytomegalovirus glycoproteins $\mathrm{gB}$ and $\mathrm{gH} / \mathrm{gL}$ mediate epithelial cell-cell fusion when expressed either in cis or in trans. J. Virol. 82, 11837-11850 (2008).

16. Rieder, F. \& Steininger, C. Cytomegalovirus vaccine: phase II clinical trial results. Clin. Microbiol. Infec. 20(Suppl 5): 95-102 (2014).

17. Wang, D. \& Fu, T. M. Progress on human cytomegalovirus vaccines for prevention of congenital infection and disease. Curr. Opin. Virol. 6, 13-23 (2014).

18. Pass, R. F. et al. A subunit cytomegalovirus vaccine based on recombinant envelope glycoprotein B and a new adjuvant. J. Infect. Dis. 180, 970-975 (1999).

19. Pass, R. F. Development and evidence for efficacy of CMV glycoprotein B vaccine with MF59 adjuvant. J. Clin. Virol. 46(Suppl 4): S73-S76 (2009).

20. Griffiths, P. D. et al. Cytomegalovirus glycoprotein-B vaccine with MF59 adjuvant in transplant recipients: a phase 2 randomised placebo-controlled trial. Lancet 377, 1256-1263 (2011).

21. Schleiss, M. R. et al. Glycoprotein B (gB) vaccines adjuvanted with AS01 or AS02 protect female guinea pigs against cytomegalovirus (CMV) viremia and offspring mortality in a CMV-challenge model. Vaccine 32, 2756-2762 (2014).

22. Navarro, D. et al. Glycoprotein B of human cytomegalovirus promotes virion penetration into cells, transmission of infection from cell to cell, and fusion of infected cells. Virology 197, 143-158 (1993).

23. Cekinovic, D. et al. Passive immunization reduces murine cytomegalovirusinduced brain pathology in newborn mice. J. Virol. 82, 12172-12180 (2008).

24. Backovic, M., Longnecker, R. \& Jardetzky, T. S. Structure of a trimeric variant of the Epstein-Barr virus glycoprotein B. Proc. Natl Acad. Sci. USA 106, 2880-2885 (2009).

25. Maurer, U. E. et al. The structure of herpesvirus fusion glycoprotein B-bilayer complex reveals the protein-membrane and lateral protein-protein interaction. Structure. 21, 1396-1405 (2013).
26. Sharma, S., Wisner, T. W., Johnson, D. C. \& Heldwein, E. E. HCMV gB shares structural and functional properties with $\mathrm{gB}$ proteins from other herpesviruses. Virology 435, 239-249 (2013).

27. Patrone, M. et al. Enhanced expression of full-length human cytomegalovirus fusion protein in non-swelling baculovirus-infected cells with a minimal fed-batch strategy. PloS ONE 9, e90753 (2014).

28. Roche, S., Bressanelli, S., Rey, F. A. \& Gaudin, Y. Crystal structure of the low-pH form of the vesicular stomatitis virus glycoprotein G. Science 313, 187-191 (2006).

29. Roche, S., Rey, F. A., Gaudin, Y. \& Bressanelli, S. Structure of the prefusion form of the vesicular stomatitis virus glycoprotein G. Science 315, 843-848 (2007).

30. Kadlec, J., Loureiro, S., Abrescia, N. G., Stuart, D. I. \& Jones, I. M. The postfusion structure of baculovirus gp64 supports a unified view of viral fusion machines. Nat. Struct. Mol. Biol. 15, 1024-1030 (2008).

31. Cairns, T. M. et al. Dissection of the antibody response against herpes simplex virus glycoproteins in naturally infected humans. J. Virol. 88, 12612-12622 (2014).

32. Ohlin, M., Sundqvist, V. A., Mach, M., Wahren, B. \& Borrebaeck, C. A. Fine specificity of the human immune response to the major neutralization epitopes expressed on cytomegalovirus gp58/116 (gB), as determined with human monoclonal antibodies. J. Virol. 67, 703-710 (1993).

33. Potzsch, S. et al. B cell repertoire analysis identifies new antigenic domains on glycoprotein B of human cytomegalovirus which are target of neutralizing antibodies. PLoS Pathog. 7, el002172 (2011).

34. Macagno, A. et al. Isolation of human monoclonal antibodies that potently neutralize human cytomegalovirus infection by targeting different epitopes on the gH/gL/UL128-131A complex. J. Virol. 84, 1005-1013 (2010).

35. Spindler, N. et al. Characterization of a discontinuous neutralizing epitope on glycoprotein B of human cytomegalovirus. J. Virol. 87, 8927-8939 (2013).

36. Wiegers, A. K., Sticht, H., Winkler, T. H., Britt \& Mach, M. Identification of a neutralizing epitope within antigenic domain 5 of glycoprotein B of human cytomegalovirus. J. Virol. 89, 361-372 (2014).

37. Spindler, N. et al. Structural basis for the recognition of human cytomegalovirus glycoprotein B by a neutralizing human antibody. PLoS Pathog. 10, e1004377 (2014).

38. Cairns, T. M. et al. Mechanism of neutralization of herpes simplex virus by antibodies directed at the fusion domain of glycoprotein B. J. Virol. 88, 2677-2689 (2014).

39. Dong, A. et al. In situ proteolysis for protein crystallization and structure determination. Nat. Methods. 4, 1019-1021 (2007).

40. Wernimont, A. \& Edwards, A. In situ proteolysis to generate crystals for structure determination: an update. PloS ONE 4, e5094 (2009).

41. Atanasiu, D., Saw, W. T., Cohen, G. H. \& Eisenberg, R. J. Cascade of events governing cell-cell fusion induced by herpes simplex virus glycoproteins $\mathrm{gD}$, gH/gL, and gB. J. Virol. 84, 12292-12299 (2010).

42. Janin, J. \& Chothia, C. The structure of protein-protein recognition sites. J. Biol. Chem. 265, 16027-16030 (1990).

43. Manley, K. et al. Human cytomegalovirus escapes a naturally occurring neutralizing antibody by incorporating it into assembling virions. Cell Host Microbe 10, 197-209 (2011).

44. Wen, Y. et al. Human cytomegalovirus gH/gL/UL128/UL130/UL131A complex elicits potently neutralizing antibodies in mice. Vaccine 32, 3796-3804 (2014)

45. Fouts, A. E., Chan, P., Stephan, J. P., Vandlen, R. \& Feierbach, B. Antibodies against the $\mathrm{gH} / \mathrm{gL} / \mathrm{UL} 128 / \mathrm{UL} 130 / \mathrm{UL} 131$ complex comprise the majority of the anti-cytomegalovirus (anti-CMV) neutralizing antibody response in CMV hyperimmune globulin. J. Virol. 86, 7444-7447 (2012).

46. Ciferri, C. et al. Structural and biochemical studies of HCMV gH/gL/gO and Pentamer reveal mutually exclusive cell entry complexes. Proc. Natl Acad. Sci. USA. 112, 1767-1772 (2015).

47. Wilson, I. A. \& Stanfield, R. L. Antibody-antigen interactions: new structures and new conformational changes. Curr. Opin. Struct. Biol. 4, 857-867 (1994).

48. Lingwood, D. et al. Structural and genetic basis for development of broadly neutralizing influenza antibodies. Nature 489, 566-570 (2012).

49. Britt, W. J., Vugler, L. \& Stephens, E. B. Induction of complement-dependent and -independent neutralizing antibodies by recombinant-derived human cytomegalovirus gp55-116 (gB). J. Virol. 62, 3309-3318 (1988).

50. Kabanova, A. et al. Antibody-driven design of a human cytomegalovirus gHgLpUL128L subunit vaccine that selectively elicits potent neutralizing antibodies. Proc. Natl Acad. Sci. USA. 111, 17965-17970 (2014).

51. Liljeroos, L., Krzyzaniak, M. A., Helenius, A. \& Butcher, S. J. Architecture of respiratory syncytial virus revealed by electron cryotomography. Proc. Natl Acad. Sci. USA. 110, 11133-11138 (2013).

52. zur Megede, J. et al. Increased expression and immunogenicity of sequencemodified human immunodeficiency virus type 1 gag gene. J. Virol. 74, 2628-2635 (2000).

53. Tang, G. et al. EMAN2: an extensible image processing suite for electron microscopy. J. Struct. Biol. 157, 38-46 (2007). 
54. van Heel, M., Harauz, G., Orlova, E. V., Schmidt, R. \& Schatz, M. A new generation of the IMAGIC image processing system. J. Struct. Biol. 116, 17-24 (1996).

55. Hohn, M. et al. SPARX, a new environment for Cryo-EM image processing. J. Struct. Biol. 157, 47-55 (2007).

56. Pettersen, E. F. et al. UCSF Chimera--a visualization system for exploratory research and analysis. J. Comput. Chem. 25, 1605-1612 (2004).

57. Collaborative Computational Project N. The CCP4 suite: programs for protein crystallography. Acta. Crystallogr. D Biol. Crystallogr. 50, 760-763 (1994).

58. McCoy, A. J. et al. Phaser crystallographic software. J. Appl. Crystallogr. 40, 658-674 (2007).

59. Bunkoczi, G. \& Read, R. J. Improvement of molecular-replacement models with Sculptor. Acta. Crystallogr. D Biol. Crystallogr. 67, 303-312 (2011).

60. Adams, P. D. et al. PHENIX: a comprehensive Python-based system for macromolecular structure solution. Acta. Crystallogr. D Biol. Crystallogr. 66, 213-221 (2010).

61. Afonine, P. V. et al. Towards automated crystallographic structure refinement with phenix.refine. Acta. Crystallogr. D Biol. Crystallogr. 68, 352-367 (2012).

62. Emsley, P. \& Cowtan, K. Coot: model-building tools for molecular graphics. Acta. Crystallogr. D. Biol. Crystallogr. 60, 2126-2132 (2004).

63. Chen, V. B. et al. MolProbity: all-atom structure validation for macromolecular crystallography. Acta. Crystallogr. D Biol. Crystallogr. 66, 12-21 (2010).

64. Bender, F. C. et al. Antigenic and mutational analyses of herpes simplex virus glycoprotein B reveal four functional regions. J. Virol. 81, 3827-3841 (2007).

\section{Acknowledgements}

We would like to thank Y. Aggarwal, C. Linton and M. Schaefer for excellent technical support, G. Palladino, S. Barnett, A. Feire and Y. Wen for insightful discussions, and M.J. Bottomley, E. Malito, I. Ferlenghi and L. Liljeroos for critically reading the manuscript.

\section{Author contributions}

S.Ch., C.C., E.C.S. and A.C. designed research; S.Ch, C.C., P.A.N., R.G., S.Ca., K.B., J.M and E.C.S. performed research; S.Ch, C.C., P.A.N, C.H., A.E.L., E.C.S. and A.C. analysed data; S.Ch and A.C. wrote the paper.

\section{Additional information}

Structural Data. Structural data for the HCMV gB-1G2 complex were deposited in the RCSB Protein Data Bank under PDB ID 5C6T.

Supplementary Information accompanies this paper at http://www.nature.com/ naturecommunications

Competing financial interests: S. Ch, S. Ca, R.G., K.B., J.M. and A.C. are employees of GlaxoSmithKline; P.A.N., E.C.S. and C.H. are employees of Novartis; C.C. is an employee of Genentech; A.E.L is an employee of Hookipa Biotech.

Reprints and permission information is available online at http://npg.nature.com/ reprintsandpermissions/

How to cite this article: Chandramouli, S. et al. Structure of HCMV glycoprotein B in the postfusion conformation bound to a neutralizing human antibody. Nat. Commun. 6:8176 doi: 10.1038/ncomms9176 (2015).

(c) (i) This work is licensed under a Creative Commons Attribution 4.0 International License. The images or other third party material in this article are included in the article's Creative Commons license, unless indicated otherwise in the credit line; if the material is not included under the Creative Commons license, users will need to obtain permission from the license holder to reproduce the material. To view a copy of this license, visit http://creativecommons.org/licenses/by/4.0/ 